\title{
Tidal dwarf galaxies as laboratories of star formation and cosmology
}

\author{
Pierre-Alain Duc, Frédéric Bournaud and Médéric Boquien ${ }^{1}$ \\ ${ }^{1}$ AIM - Unité Mixte de Recherche CEA - CNRS - Université Paris VII - UMR nº 7158 Service \\ d'Astrophysique, CEA-Saclay, 91191 Gif-sur-Yvette, France \\ email: paduc@cea.fr
}

\begin{abstract}
Star formation may take place in a variety of locations in interacting systems: in the dense core of mergers, in the shock regions at the interface of the colliding galaxies and even within the tidal debris expelled into the intergalactic medium. Along tidal tails, objects may be formed with masses ranging from those of super-star clusters to dwarf galaxies: the so-called Tidal Dwarf Galaxies (TDGs). Based on a set of multi-wavelength observations and extensive numerical simulations, we show how TDGs may simultaneously be used as laboratories to study the process of star-formation (SFE, IMF) in a specific environment and as probes of various cosmological properties, such as the distribution of dark matter and satellites around galaxies.
\end{abstract}

Keywords. galaxies: dwarf, galaxies: starburst,galaxies: formation,galaxies: interactions

\section{The various observed types of star-forming tidal objects}

Star-formation in colliding galaxies has mostly been studied in their inner most regions. There the accumulation of gas, previously funneled by various dynamical processes, and its further collapse may lead to intense circum-nuclear starbursts. A mode of spatially extended, probably shock-induced, star-formation has also been observed for a long time at the interface of the interacting galaxies, but was only recently modeled (Barnes 2004). Further out the detection of HII regions along tidal tails proved that stars may form well outside the disks of the parent galaxies. The puzzling discovery of even more distant star-forming regions, located in the intergalactic medium, at more than $100 \mathrm{kpc}$ from any massive galaxy, has raised the question of their origin. Given these large distances and related timescales, the young intergalactic stars were born in situ. The relatively high metallicity measured in the associated ionized gas (typically half solar to solar) indicate that, without any doubt, the gas fueling these intergalactic SF episodes had previously been pre-enriched in the disk of parent galaxies and had later been expelled by a dynamical process: tidal forces or, for systems belonging to dense groups or clusters, by ram-pressure.

This paper focusses here on the formation of stars and stellar systems in the outermost regions of colliding galaxies. Before presenting how star-formation may take place far from the parent galaxies, it is worthwhile to briefly enumerate the various types of external star-forming objects so-far described in the literature:

- Intergalactic emission-line regions, with optical spectra typical of star-forming HII regions. They usually consist of small, often compact - they are also refereed as ELDots - blue condensations made of a few OB stars. Their Star Formation Rates are below $0.01 \mathrm{M}_{\odot} \mathrm{yr}^{-1}$ (Gerhard et al. 2002, Ryan-Weber et al. 2004, Mendes de Oliveira et al. 2004, Cortese et al. 2006). They will probably not form gravitationally bound objects but may contribute to the population of Intergalactic Stars, recently found in nearby clusters of galaxies. 


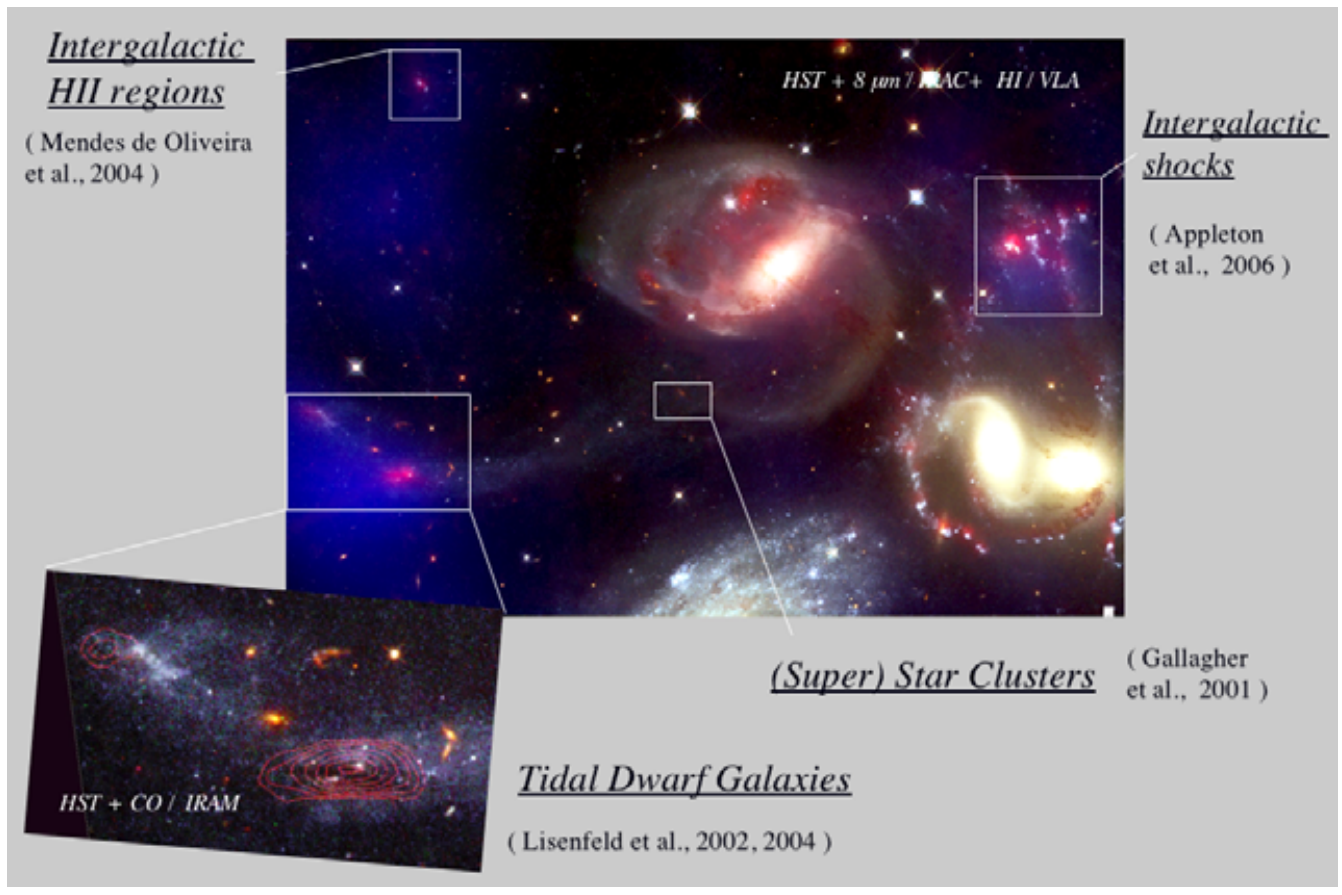

Figure 1. Various modes of Intergalactic Star Formation in the Stephan's Quintet. The main image is a montage of the Spitzer/IRAC $8 \mu \mathrm{m}$ emission (in red), tracing the star-forming regions, the VLA HI emission (in blue), showing the gas reservoirs, superimposed on an optical HST image, showing the stellar populations and dust lanes

- Young (Super) Star clusters, born in giant HII complexes and located along tidal tails (e.g., Weilbacher et al. 2003, de Grijs et al. 2003, López-Sánchez et al. 2004). Having typical typical masses of $10^{6-7} \mathrm{M}_{\odot}$, the latter may evolve into Globular Clusters (e.g. Schweizer et al. 1996).

- Tidal Dwarf Galaxies, i.e. star-forming, gravitationally bound, objects made of tidal material, with apparent masses and sizes of dwarf galaxies. The most massive of them exceed $10^{9} \mathrm{M}_{\odot}$ and are usually found close to the end of tidal tails. They contain large quantities of gas in atomic, molecular and ionized form (Braine et al. 2001).

The variety of external star-forming regions/objects is perfectly illustrated by the Stephan's Quintet (HCG92), a well studied compact group of galaxies (see Figure 1). Instances of intergalactic HII regions (Mendes de Oliveira et al. 2004), young Star Clusters along tidal tails (Gallagher et al. 2001), Tidal Dwarf Galaxies (Lisenfeld et al. 2004) and even, probably shock induced Star Formation (Appleton et al. 2006), were already reported in the intragroup medium of HCG92.

\section{The formation and evolution of sub-structures along tidal tails, according to simulations}

Depending on the characteristics of the star-forming regions, various stellar objects will or will not be formed in tidal debris. Numerical simulations may help in understanding the origin and the evolution of sub-structures along tidal tails.

Soon after the study by Mirabel et al. (1992) of a TDG candidate in the Antennae system, Barnes \& Hernquist (1992) and Elmegreen et al. (1993) published numerical models exhibiting bound condensations along tidal tails with typical masses of $10^{6-8} \mathrm{M}_{\odot}$. 

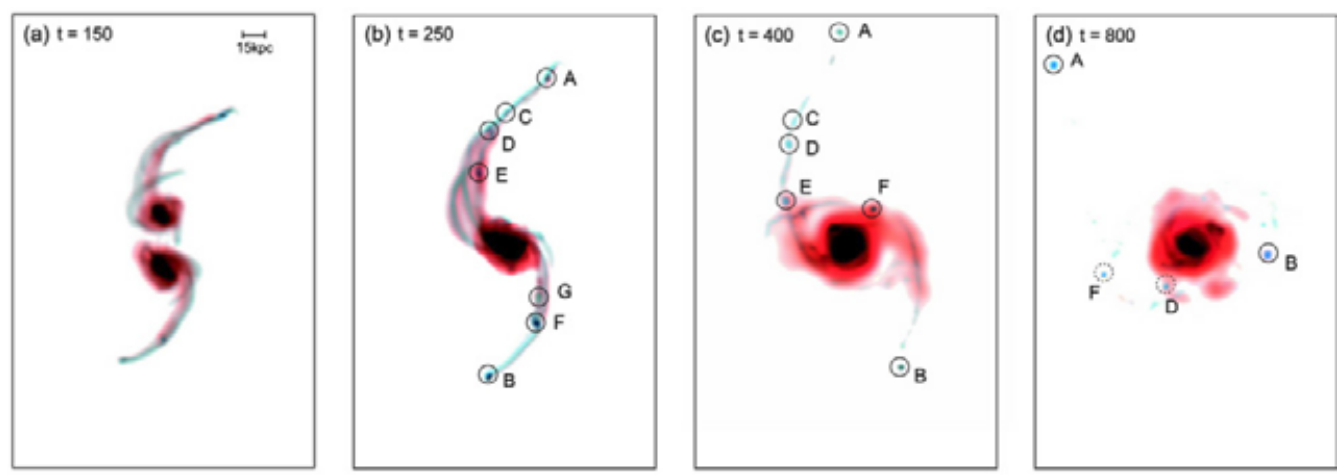

Figure 2. Numerical simulation of colliding galaxies. On each snap shot, the surviving tidal objects are identified (adapted from Bournaud \& Duc, 2006).

While in the Barnes \& Hernquist (1992) simulations, the condensations formed from gravitational instabilities in the stellar component, those of Elmegreen et al. (1993) were produced from gas clouds, the velocity dispersion of which had increased due to the collision. The direct formation of bound stellar objects in models with limited resolution has been questioned by Wetzstein et al. (2006). On the other hand, they claimed that the gaseous condensations themselves were not numerical artifacts.

For practical reasons, most simulations of galaxy-galaxy collisions assume that the dark matter halo around the parent galaxies is truncated, since, at first approximation, the latter does not play a major role in the shaping of tidal tails. However, Bournaud et al. (2003) found that the internal structure of tidal tails does actually depend on the size of the DM halo. When the latter exceeds ten times the size of the optical disk, prominent concentrations of mostly gaseous matter, with masses exceeding $10^{9} \mathrm{M}_{\odot}$, may form near the end of the tails. Such accumulations of gas resemble those present in the HI maps of several interacting systems. Duc et al. (2004) further explained that the shape of tidal forces, within the potential well of an extended DM halo, were such that gas clouds, originally located in the outskirts of the parent galaxies, can be pulled out far away from them without being diluted. In the truncated DM halo case, matter is stretched along the tails, preventing the formation of massive condensations. One should note that the presence of extended DM haloes is a prediction of cosmological simulations which has had sofar only a few rather indirect observational confirmations. However, having an extended DM halo is a necessary but not sufficient condition to form massive tidal objects. As shown by Bournaud \& Duc (2006), favorable geometrical parameters for the collision and the initial presence of extended gaseous disks are also key ingredients. The mass ratio between the parent galaxies, which should not be too different, does also matter.

Therefore, depending on their initial conditions, simulations predict the formation along tidal tails of a variety of objects with different origins: local instabilities along tidal tails, forming low or intermediate mass bound objects; a kinematic origin for the most massive ones. How these different types of objects evolve, how long they survive as independent bodies, are key questions that have sofar not had any observational answer, but may again be addressed with numerical simulations.

Based on the analysis of about $100 \mathrm{~N}$-body simulations and 600 tidal objects (see one example on Figure 2), Bournaud \& Duc (2006) found that, while most of the intermediate mass tidal objects along the tails vanished in a few $10^{8}$ yrs, those initially located near the end of the tails, with typical masses of $10^{9} \mathrm{yrs}$, could survive up to at least 
2 Gyr. They orbit around their parent galaxies like satellite galaxies. The projected distribution of the long-lived tidal objects turns out to be remarkably similar to that of the SDSS satellites around their host (Yang et al. 2006). In particular they show a similar anisotropy; the latter has probably a cosmological origin, but at least part of it could be due to a contamination by a population of Tidal Dwarf Galaxies. The simulations by Bournaud \& Duc (2006) did not have enough resolution to study the fate of the lowestmass tidal objects. According to the ad-hoc simulations by Kroupa (1997), those suffer severe gravitational evaporation, loose a significant fraction of their mass, but some of them could still survive as low-mass dwarf spheroidals. Other survivors may turn out to be the progenitors of the Super Star Clusters or even the Young Globular Clusters which were discovered in interacting systems.

\section{Physical properties of intergalactic star-forming regions}

As shown before, Star Formation in interacting systems may occur in a large variety of environments from the densest ones to the most diffuse ones. It could be expected that the modes of SF and thus the Initial Mass Function (IMF) or the Star Formation Efficiency (SFE) would change from one type of regions to the other. Surprisingly, Gao \& Solomon (2004) recently claimed that the SFE may not be so different in the dense core of Ultraluminous Infrared Galaxies and in the more quiescent regions of spiral arms. This result urges the comparison with the other extreme: the outermost regions of spirals and intergalactic star-forming regions.

We have studied the Star Formation processes in the latter environment combining three popular indicators: the ultraviolet emission obtained with Galex, which is sensitive to the SF episodes of time scales of about $100 \mathrm{Myr}$, the $\mathrm{H} \alpha$ emission, probing SF over time scales of less than $10 \mathrm{Myr}$ and finally the mid-infrared emission obtained with Spitzer, which has a slightly higher timescale. Putting together the values of the Star Formation Rates estimated with each of these tracers, and comparing them with the amount of gas reservoirs, one may determine the dust obscuration and hence the total SFR and SFE as well as speculate on the starburst ages. The IMF and recent star formation history can further be reconstructed from the analysis of the full Spectral Energy Diagram.

The first systematic studies of colliding galaxies with Spitzer indicate that globally the level of external star-formation, in particular along tidal tails, is rather low, contributing for less than $10 \%$ to the total SFR (Struck et al. in this symposium). The objects we have selected, on the contrary, exhibit in their surroundings collisional debris that are particularly active. For instance, in the interacting system NGC 5291 (see figure 3 and Boquien et al., in these proceedings), more than $80 \%$ of the current Star Formation occurs outside the main galaxies, in about 30 individual intergalactic HII regions aligned along a huge $150-\mathrm{kpc}$ long HI ring-like structure. The two most luminous ones have properties similar to TDGs, as defined in the previous section.

\section{Conclusions: TDGs as laboratories of star formation and cosmology}

Intergalactic SF regions, in general, Tidal Dwarf Galaxies, in particular, appear as attractive laboratories to study the processes of star formation. Indeed, on one hand, they share with galactic SF regions the chemical characteristics - at first order, they have the same ISM, including the molecular gas found in quantity in tidal tails -; on the other hand, they are by nature detached and isolated and are thus much simple to study. Indeed, many of the observed intergalactic star forming regions were formed within 

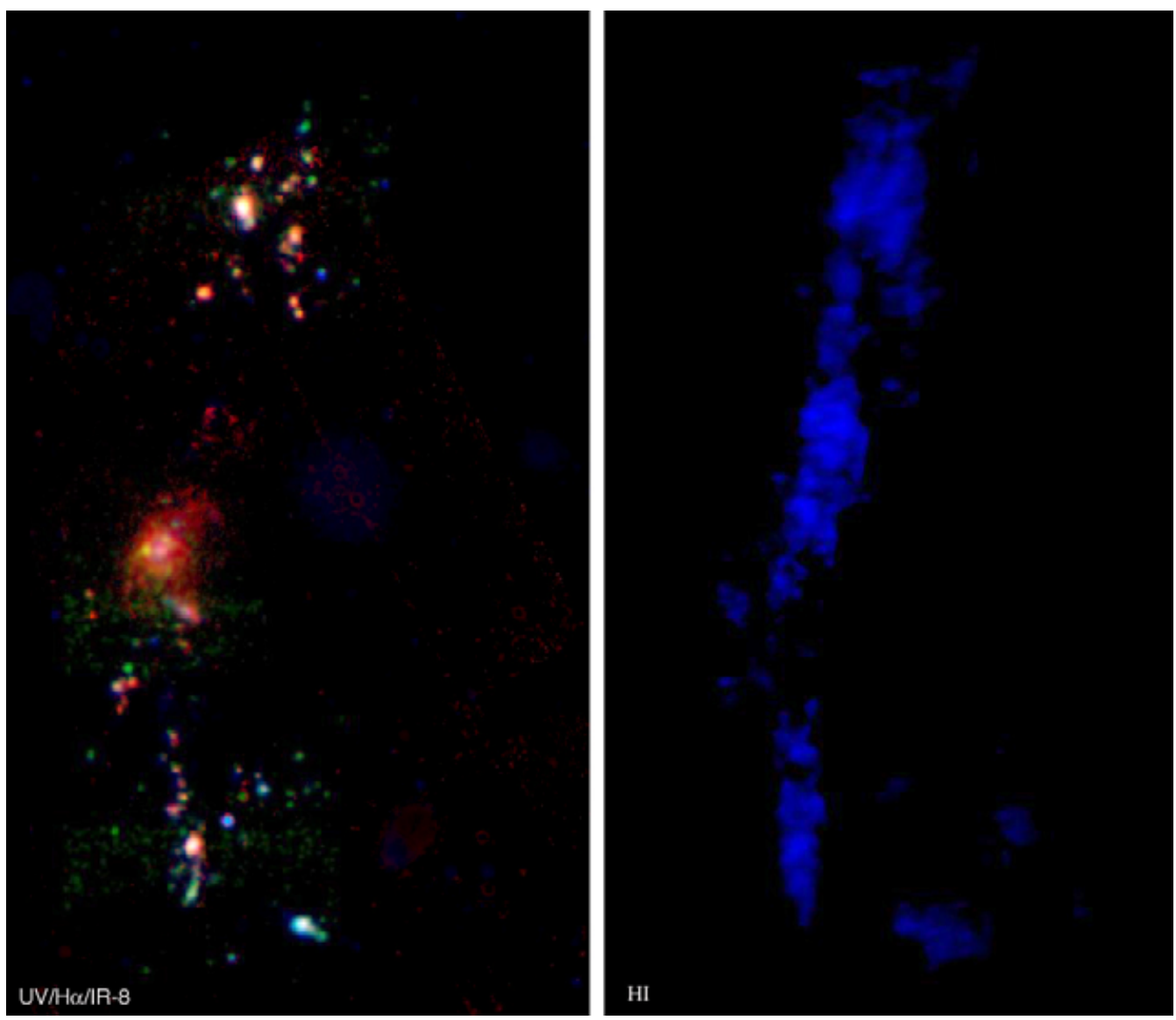

Figure 3. Left: composite image of the NGC 5291 system in pseudo-colours: near-ultraviolet Galex (blue), $\mathrm{H} \alpha$ (green) and Spitzer/IRAC $8 \mu \mathrm{m}$ (red), i.e. a combination of three starformation indicators. Right: VLA-B array HI map with the same scale. The 30 intergalactic star-forming regions along the HI-ring like structure are characterized by a UV excess, best explained by fading, quasi-instantaneous starbursts ignited less than $10 \mathrm{Myr}$ ago (Boquien et al., in prep.). No evidence for an old stellar population has yet been found.

"pure" expelled gas clouds, with no evidence for the presence of a pre-existing stellar component from the parent galaxies. Without such a contamination, the star-formation history ( $\mathrm{SFH}$ ) can be reconstructed with simple Single Star Population models. In galactic disks where several generations of stars coexist, deriving the SFH is much more complex and ambiguous. Beside, for those SF episodes triggered by a tidal interaction, numerical simulations may provide dynamical ages for the system and thus upper limits on the SF onset time, provided it occurred after the formation and development of tidal tails.

Moreover, Tidal Dwarf Galaxies may be used to constrain some parameters related to CDM cosmology, even-though these second-generation galaxies should not contribute much more than $10 \%$ to the overall dwarf galaxy population. First of all, TDGs with masses exceeding $10^{9} \mathrm{M}_{\odot}$ may only be formed if their parent galaxies were surrounded by an extended halo of dark matter, or more precisely if they were within a potential well causing flat rotation curves. Cosmological simulations predict that the halos made of nonbaryonic DM should be large; however their real sizes are difficult to measure directly. Second, because TDGs are made of material initially located in the disks of their parent 
galaxies, they should contain little quantities of the halo-type non baryonic dark-matter. Thus, measuring their dynamical mass, and comparing it with the luminous one, should reveal the presence or absence of a yet unknown, baryonic, component of dark matter located in spiral disks. This would be a direct test for the existence of large quantities of, for instance, very cold otherwise undetectable molecular clouds. Obviously deriving dynamical masses in objects as complex and young as tidal tails is still a challenge. This is nevertheless feasible in the most nearby systems (Braine et al., 2006, Bournaud et al., 2006 in prep.). Finally, numerical simulations predict that the long-lived TDGs have orbits resembling those of satellite galaxies around their host. The number and distribution of the latter are critical as they strongly constrain the cosmological, hierarchical, models. The origin for the apparent differences between the observed and predicted numbers of satellites has been actively debated for years. The existence of an additive population of dwarfs of tidal origin may even increase the discrepancies.

One should keep in mind that many of the above conclusions relied on numerical simulations that, even-though they were thoroughly checked (in particular using several codes and numerical resolutions), should be confirmed with observations. So far, only young, still forming, Tidal Dwarf Galaxies have been found and studied. They were classified as such thanks to the tidal tail linking them to their parent galaxies. Older detached TDGs are obviously much more difficult to pinpoint; unambiguous examples of such long-lived objects are still to be found. The absence of a dominating dark matter halo, an unusually high metallicity, are two hints for a tidal origin, which may be checked in nearby objects.

\section{References}

Appleton, P. N., et al. 2006, ApJ 639, L51

Barnes, J. E. \& Hernquist, L. 1992, Nature 360, 715

Barnes, J. E. 2004, MNRAS 350, 798

Bournaud, F., Duc, P.-A. \& Masset, F. 2003, A\&A 411, L469

Bournaud, F. \& Duc, P.-A. 2006, A\&\&A 456, 481

Braine, J., Duc, P.-A., Lisenfeld, U., et al. 2001, A\&A 378, 51

Cortese, L., Gavazzi, G., Boselli, A., et al. 2006, A\&A 453, 847

de Grijs, R., Lee, J. T., Clemencia Mora Herrera, M., et al. 2003, New Astronomy 8, 155

Duc, P.-A., Bournaud, F. \& Masset, F. 2004, A $\mathscr{\mho} A$ 427, 803

Elmegreen, B. G., Kaufman, M. \& Thomasson, M. 1993, ApJ 412, 90

Gallagher, S. C., Charlton, J. C., Hunsberger, S. D., Zaritsky, D. \& Whitmore, B. C. 2001, AJ 122,163

Gao, Y. \& Solomon, P. M. 2004, ApJ 606, 271

Gerhard, O., Arnaboldi, M., Freeman, K. C. \& Okamura, S. 2002, ApJ 580, L121

Kroupa, P. 1997, New Astronomy 2, 139

Lisenfeld, U., Braine, J., Duc, P.-A., Brinks, E., Charmandaris, V. \& Leon, S. 2004, A\&\&A 426, 471

López-Sánchez, Á. R., Esteban, C. \& Rodríguez, M. 2004, ApJS 153, 243

Mendes de Oliveira, C., Cypriano, E. S., Sodré, L. \& Balkowski, C. 2004, ApJ 605, L17

Mirabel, I. F., Dottori, H. \& Lutz, D. 1992, A\&A 256, L19

Ryan-Weber, E. V., Meurer, G. R., Freeman, K. C., et al. 2004, AJ 127, 1431

Schweizer, F., Miller, B. W. \& Whitmore, B. C. \& Fall, S. M. 1996, AJ 112, 1839

Weilbacher, P. M., Duc, P.-A. \& Fritze-v. Alvensleben, U. 2003, A\&A 397, 545

Wetzstein, M., Naab, T. \& Burkert, A. 2006, astro-ph/0510821

Yang, X., van den Bosch, F. C., Mo, H. J., et al. 2006, MNRAS 528 


\section{Discussion}

TAN: Star formation synchronized to 10 Myr along a tidal tail that takes $\sim 100$ Myr to form is surprising. Do our (or other's) numerical simulations of star formation in tidal tails predict this?

Duc: The time scale to form the tidal tails is about 100-200 Myr. This provides an upper limit for the age of the starbursts ignited in the HI structure. There is besides no hint for an old stellar population along the tail, either produced locally or expelled from the parent galaxies. Within the intergalactic medium, they should be easily detectable.

Whitmore: The finding that the clusters are $<10 \mathrm{Myr}$ is almost certainly another example of the infant mortality of clusters I talked about this morning rather than the very improbably chance that you are suddenly having a simultaneous burst of young clusters all along the tail some hundred Myr after the initial tidal interaction that pulled the tails out. Whenever you have roughly constant star formation (e.g., spirals), you will always find that the average age is $<10 \mathrm{Myr}$, since $90 \%$ of clusters dissolve each decade of time.

Duc: The star-forming regions studied in this paper were not identified based on the presence of star clusters (possibly suffering a strong infant mortality), but were rather traced by younger HII regions. I nevertheless agree that it may be intriguing to have a quasi-simultaneous onset of star-formation along an HI structure as long as $150 \mathrm{kpc}$, like in NGC 5291. However, one should note that, for this specific object, the star-formation time scale is not dramatically different than the dynamical time scale for the formation of the HI collisional ring: up to $300 \mathrm{Myr}$, according to numerical simulations. Besides, in the case of a bulls-eye collision, the star-formation along the ring cannot start just after the impact, unlike in tidal tails. An additional period is required so that the gas can (re)condense. On the observational side, no hint for an old stellar population has yet been found. Given the nature of the object, mostly lying in the intergalactic medium, stars older than 100 Myr should be quite easy to detect.

MirABEL: You find that dark matter must be large to reproduce the observations. What can you say about baryonic versus non-baryonic dark matter?

Duc: While simulations show that TDGs should contain only a tiny amount of dark matter particles from the cosmological halos, they may have acquired baryonic dark matter from the disk of their parents. Measuring the dynamical mass of TDGs and comparing it with the luminous mass may hence tell about the DM content in the disks of spirals. Our initial measures indicate no strong evidence for large quantities of baryonic DM there. However, observational errors are still large and this result needs to be confirmed.

OEY: I'd like to draw attention to the poster S237.681 by Werk et al.in which she has identified some intergalactic HII regions. Her work raises the question of whether the Schmidt law and Kennicutt threshold applies. I realize you don't have CO data for all of your objects, but wonder whether you have any impressions on this issue.

DuC: Unfortunately, we do not have enough objects for which we could map the CO and thus probe the Kennicutt law in that special environment.

STRUCK: Not all tails have dwarfs at the end. If your theory is right, maybe the range of tail types can tell us about the range of halo properties? 
Duc: Indeed, this is a promising method. However, other parameters should be taken into account. Having an extended dark matter halo is required to form TDGs, but this is not sufficient. The initial size of the gaseous disk in the parent galaxies, the geometrical parameters of the encounter, the mass ratios of the colliding galaxies are also key parameters. 